\title{
High mixing performances of shear-thinning fluids in two-layer crossing channels micromixer at very low Reynolds numbers
}

\author{
A. Kouadri ${ }^{1,2^{*}}$, Y. Lasbet ${ }^{2}$ and M. Makhlouf ${ }^{1}$ \\ ${ }^{1}$ Department of Mechanical Engineering, Djillali Liabes University, \\ 22000 Sidi Bel Abbes, Algeria \\ ${ }^{2}$ LDMM Laboratory, Djelfa University, 17000 Djelfa, Algeria \\ *Email : kouadriamar@yahoo.fr \\ Phone : +213698968998
}

\begin{abstract}
In a recent study, the Two-Layer Crossing Channels Micromixer (TLCCM) exhibited good mixing capacities in the case of the Newtonian fluids (close to 100\%) for all considered Reynolds number values. However, since the majority of the used fluids in the industrial sectors are non-Newtonians, this work details the mixing evolution of power-law fluids in the considered geometry. In this paper, the power-law index ranges from 0.73 to 1 and the generalized Reynolds number is bounded between 0.1 and 50. The conservation equations of momentum, mass and species transport are numerically solved using a CFD code, considering the species transport model. The flow structure at the cross-sectional planes of our micromixer was studied using the dynamic systems theory. The evolutions of the intensity, also the axial, radial and tangential velocity profiles were examined for different values of the Reynolds number and the power-law index. Besides, the pressure drop of the power-law fluids under different Reynolds number was calculated and represented. Furthermore, the mixing efficiency is evaluated by the computation of the mixing index (MI), based on the standard deviation of the mass fraction in different cross-sections. In such geometry, a perfect mixing is achieved with MI closed to $99.47 \%$, at very small Reynolds number (from the value 0.1) whatever the power-law index and generalized Reynolds numbers taken in this investigation. Consequently, the targeted channel presents a useful tool for pertinent mass transfer improvements, it is highly recommended to include it in various microfluidic systems.
\end{abstract}

Keywords: Micromixer; chaotic advection; mixing index; shear-thinning fluids; low Reynolds number.

\section{INTRODUCTION}

The flow at low Reynolds number and small scales are necessarily laminar where the particle trajectories are regular. So, this type of flows is unfavourable for achieving a perfect and rapid mixing in the transfer phenomena (heat and mass) prevailing in the ducts. To overcome this difficulty, we had to disturb the flow, either spatially or temporally, in order to affect the trajectories of fluid particles from regular to chaotic. This task becomes more complicated in 
the case of creeping flows (Re of the order of 0.1 ). Temporary perturbation concerns twodimensional flows where solid boundaries and/or initial conditions are disturbed over time. Regarding the spatial perturbation, it makes the geometry twisted in the three directions of space. These perturbations break the flow regularity and so the chaotic flow is generated. For this subject, it became necessary to focus the efforts on the development of micromixers used in the different industrial domains such as bioengineering and chemical engineering, chemical synthesis, emulsion processes, polymerization [1], DNA analysis, two-phase flow separation [2], detection and analysis of chemical or biochemical content [3-4-5]. We can distinguish two main types of micromixers: active and passive micro-mixers [6]. Active micromixers use external energy sources to accentuate the flow agitation by, for example, a magnetic field, pressure field, and electrical field, etc. These types of micromixers are very efficient in terms of mixing [7] but they are more complex and expensive than passive micromixers. In addition, they required more detailed in terms of fabrication. Besides, passive micromixers are of great importance due to their simple structures and easy manufacturing [8]. Thus, the secondary flows resulting from the chaotic advection are very intense and many vortices of different sizes are generated. The large vortices improve the macroscopic mixing against the small ones act microscopically on the homogenization. All these reasons encourage researchers to focus their efforts on the design and realization of new micromixers operating in chaotic advection especially in the case where the working fluid is a non-Newtonian fluid because it is the most used in the industry. Niederkorn and Ottino [9] characterized the mixing of non-Newtonian fluids that was modelled by Carreau model in a 2D periodic flow between two eccentric cylinders. The authors showed that the shear thinning behaviour of the fluid decreases the velocity of the flow so consequently the mixing was extended. The velocity of flow is also extended in 2D and 3D cavities stirred periodically where the Carreau model is adopted by Anderson et al. [10]. Chao et al. [11] carried out a numerical investigation of the mixing of non-Newtonian shear-thinning and shear-thickening fluids driven electrokinetically through microchannels containing rectangular and wavy blocks. They studied the impact of diverse parameters on the mixing efficiency like the power-law index, the wave amplitude, the width and length of the blocks, and the applied electric field potential. The results indicated that for both types of fluid, the mixing performance improved by increasing the amplitude of the wave, increasing the length and width of the blocks, and increasing the magnitude of the heterogeneous surface potential.

Afzal and Kim [12] performed a numerical simulation to investigate the mixing behavior of Newtonian (water) and non-Newtonian (blood) fluids through T-shaped right and serpentine microchannels. They found that the serpentine channel showed higher mixing performance for all flow regimes. Tsai et al. [13] studied the mixing evolution of Newtonian fluid (deionized water (DI)) and non-Newtonian fluid carboxymethyl cellulose (CMC) solutions in three serpentine-type micromixers composed of repeated $\mathrm{C}$-shaped units. They outlined that the mixing efficiency of a micromixer with a lower centerline radius is better compared to that obtained for a larger centerline radius. So, the curvature-induced vortices improve considerably fluid mixing. A numerical study was checked out by Shamsoddini et al. [14] in order to investigate the effects of the power-law index and stirrer frequency on the mixing of non-Newtonian fluids in an active micromixer using an oscillating stir-bar. They considered two Reynolds numbers, 20 and 72. Their results showed that this active micromixer is not useful for shear-thickening fluids. While for the shear-thinning fluids the 
mixing is more efficient and fast. Therefore, the mixing index increased steadily with the increase of the power-law indices.

Islami, Khezerloo, and Gharraei [15] carried out a numerical study on the mixing of shear-thinning fluids, using six models of curved micromixers for a wide range of the Reynolds numbers with different values of power-law indices. The mixing index decreased with the decrease of the power-law index. Recently, Islami and Khezerloo [16] studied numerically the mixing of non-Newtonian shear-thinning fluids in a curved micromixer with grooves integrated on the bottom wall surface. They showed that the grooves revealed the generation of chaotic advection and the enhancement of the mixing performance where the $30^{0}$ is the optimal orientation. Xia et al. [17], employed micromixer for Newtonian fluids providing good mixing at low Reynolds numbers. The micromixer consisted of two-layer crossing channels, together with numerical and experimental investigations show that the micromixer is more efficient, which showed excellent mixing performance over a wide range of Reynolds numbers. A mixing index of 0.96 was obtained for $\mathrm{Re}=0.2$. In a recent paper, Hossain et al. [18] characterized the mixing performance of Newtonian fluids in two-layer crossing channels micromixer for Reynolds number ranging from 0.2 to 40 . Their results outline that the mixing index reached 0.90 with variations of the geometric parameters even at creeping flow, $\mathrm{Re}=0.2$.

Thereafter Hossain et al. [19], invented an improved geometry model that can achieve a higher mixing index of 0.99 with Reynolds number less than 10 , the superiority of the micromixer has been demonstrated both numerically and experimentally.

The purpose of this study is to highlight the performance of the geometry proposed by Hossain et al [19] in terms of mixing in the case of non-Newtonian pseudo plastic fluids after having proved his great abilities to achieve a perfect mixing of Newtonian fluids. Knowing that the literature review showed that the mixing index obtained at low Reynolds number ( 0.1 to 50$)$ in the various proposed systems does not exceed $50 \%$ in the best of cases. Analyses of the mixing process are done by using the CFD numerical tool in the case of power-law fluids, carboxymethyl cellulose (CMC) solutions. The power-law index ranging from 0.73 to 1 and the generalized Reynolds number varies from 0.1 to 50 .

\section{GEOMETRY OF THE MICROMIXER}

The main geometry adopted in our study is called TLCCM and it is detailed in Figure 1. It is initially proposed by Hossain et al. [19]. This micromixer is composed of two twisted channels of identical dimensions, the upper and lower channels are arranged periodically. The fluids in channels are interconnected through the mediums of the shapes " $\mathrm{X}$ " and vertical sections. The successive arrangement of the mixing units results in repetitive patterns of elongation, fragmentation, and recombination of the fluid streams. The dimensions of the micromixer geometry are presented on Figure 1, where the width of the micromixer $\mathrm{H}$ is equal to $1.07 \mathrm{~mm}$, the width of the diagonal channel $\mathrm{W}=0.3 \mathrm{~mm}$, the length of the vertical channel $b=0.15 \mathrm{~mm}$, the pitch between the transverse channels $\mathrm{P}=0.64 \mathrm{~mm}$, the height of diagonal channel $\mathrm{d}=0.15 \mathrm{~mm}$ and the number of mixing units is equal to 9 . The total length of the micromixer is $7.5 \mathrm{~mm}$; the cross-sections of the inlets and outlet are $0.15 \times 0.3 \mathrm{~mm}^{2}$ and $0.3 \times 0.3 \mathrm{~mm}^{2}$ respectively. 


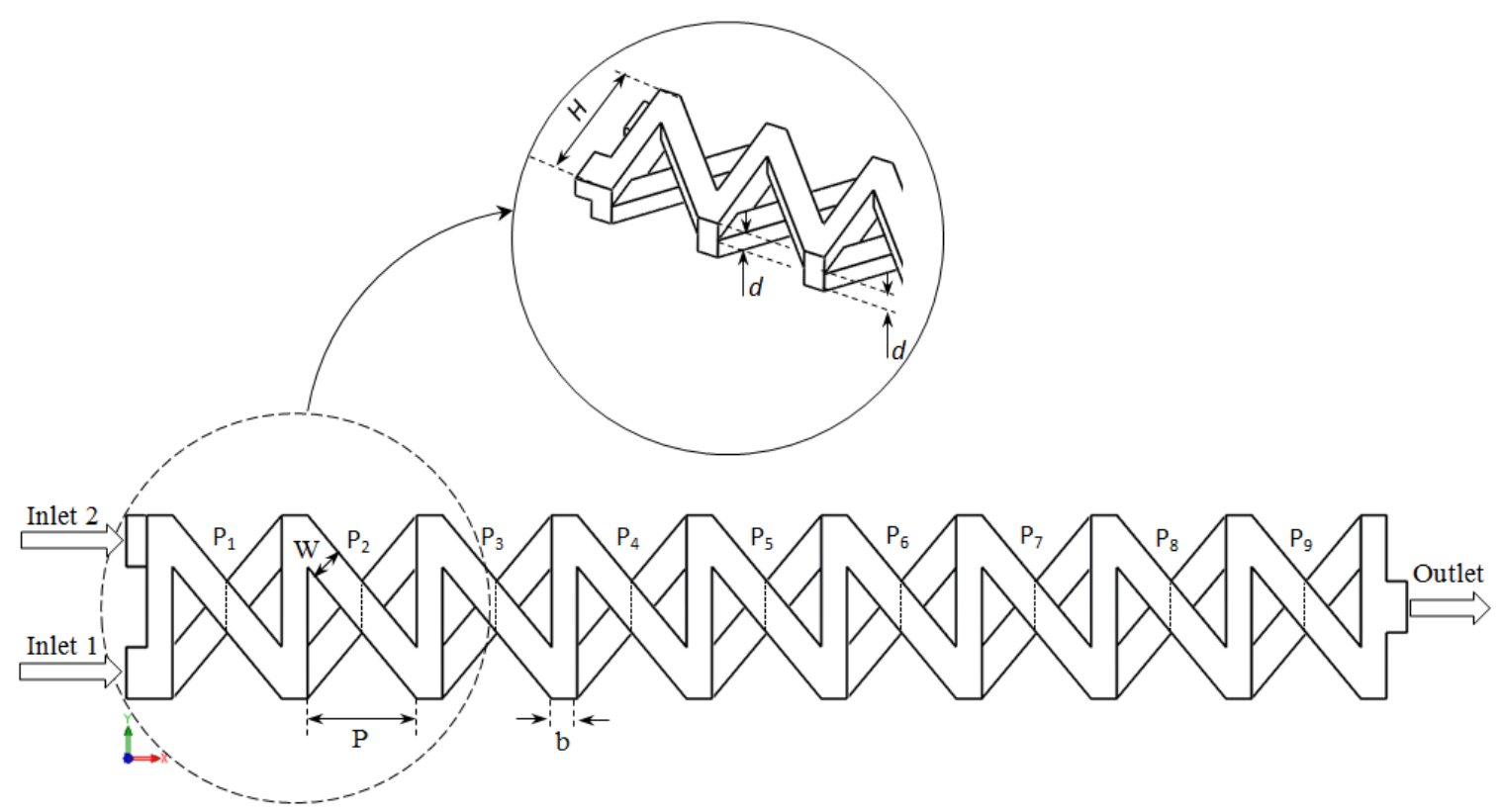

Figure 1. Description of the TLCCM micromixer.

\section{MATHEMATICAL AND NUMERICAL MODELING}

\section{Governing Equations}

Steady conservation equations of incompressible fluid are solved numerically by using a CFD code. These equations can be expressed as follows:

$$
\begin{aligned}
& \nabla \cdot \mathrm{V}=0 \\
& \rho(\mathrm{V} \cdot \nabla) \mathrm{V}=-\nabla \mathrm{P}+\nabla \cdot \overline{\bar{\tau}}
\end{aligned}
$$

where $\mathrm{V}, \rho, \mathrm{P}$ and $\overline{\bar{\tau}}$ represent the velocity, fluid density, static pressure, and the shear stress tensor, respectively.

The species transport equation is given by Equation. (3):

$$
(\mathrm{V} \cdot \nabla) \mathrm{C}=\mathrm{D} \nabla^{2} \mathrm{C}
$$

where $\mathrm{D}$ is the diffusion coefficient and $\mathrm{C}$ is the concentration.

A generalized form of the shear stress tensor is written under the following form, see Equation. (4):

$$
\overline{\bar{\tau}}=\mu_{\mathrm{a}} \overline{\bar{\gamma}}
$$

With $\overline{\bar{\gamma}}$ is the shear rate tensor, see Equation. (5):

$$
\overline{\dot{\gamma}}=\frac{1}{2}\left(\nabla \mathrm{V}+\nabla \mathrm{V}^{\mathrm{T}}\right)
$$

Exponent $\mathrm{T}$ indicates the transposed operator.

In our study, the power-law viscosity model was used to study the rheological behavior of CMC solutions. In this model, the apparent viscosity $\mu_{\mathrm{a}}$ is calculated as:

$$
\mu_{\mathrm{a}}=m \dot{\gamma}^{\mathrm{n}-1}
$$

where $\mathrm{m}$ denotes the consistency coefficient and $\mathrm{n}$ denotes the power-law index of the fluids. Shear rate $\dot{\gamma}$ is defined as: 


$$
\dot{\gamma}=\sqrt{1 / 2(\overline{\bar{\gamma}}: \overline{\bar{\gamma}})}
$$

As boundary conditions, no-slip velocity condition is imposed at the wall, a constant uniform velocity is imposed at the inlets cross-section and pressure outlet condition is established at the outlet section. The mass fraction at the inlet 1 equals to 0 while in the inlet 2 is equal to 1 .

The Semi-Implicit Method for Pressure Linked Equations-Consistent (SIMPLEC) algorithm was used to solve the pressure-velocity coupled equations. A high resolution second-order upwind scheme was used for the discretization of the momentum equations as well as the species transport equation. The convergence criterion in all simulations is taken less than $10^{-6}$.

\section{Physical Properties of Fluids}

In this work, the working fluid is CMC solutions. The density of CMC solutions (Srisamran et al., [20]) used in this work was $1000 \mathrm{~kg} / \mathrm{m}^{3}$, the consistency coefficient and the power-law index of CMC solutions are listed in Table 1 (Fellouah et al., [21], Whitelaw et al., [22]). The diffusion coefficient of the CMC solutions was assumed to be $3.6 \times 10^{-10} \mathrm{~m}^{2} / \mathrm{s}$ (Das et al., [23]). This value is chosen so that the molecular diffusion is negligible, so, to highlight the chaotic advection.

Table 1. Properties of fluids [21,22].

\begin{tabular}{ccc}
\hline CMC (\%) & n (-) & m (Pa.s $\left.\mathbf{s}^{\mathbf{n}}\right)$ \\
\hline $\mathbf{0}$ & 1 & 0.000902 \\
$\mathbf{0 . 1}$ & 0.9 & 0.0075 \\
$\mathbf{0 . 2}$ & 0.8 & 0.06 \\
$\mathbf{0 . 3}$ & 0.73 & 0.15 \\
\hline
\end{tabular}

To quantify the mixing performance of the micromixer, the mixing index (MI) in each crosssectional plane perpendicular to the flow direction is calculated as follows:

$$
\mathrm{MI}=1-\frac{\sigma}{\sigma_{0}}
$$

$\sigma$ represents the standard deviation of the mass fraction at cross-section defined as:

$$
\sigma^{2}=\frac{1}{N} \sum_{i=1}^{N}\left(c_{i}-\bar{c}\right)^{2}
$$

where $\sigma_{0}$ is the standard deviation at the inlet section, $\mathrm{N}$ denotes the total number of sampling points in transversal cross-section, $\mathrm{c}_{\mathrm{i}}$ is the mass fraction at a sampling point $\mathrm{i}$, and $\overline{\mathrm{c}}$ is the mean mixing mass fraction of $c_{i}$ and it is equal to 0.5 . For unmixed fluids, $M I=0$ and for a perfect mixing $\mathrm{MI}=1$.

The generalized Reynolds number $\mathrm{Re}_{\mathrm{g}}$ for a power-law fluids flow model through rectangular cross section for different aspect ratios is defined by Metzner and Reed relation [24], see Equation. (10): 


$$
\operatorname{Re}_{\mathrm{g}}=\frac{\rho \overline{\mathrm{u}}^{2-\mathrm{n}} \mathrm{d}_{\mathrm{h}}^{\mathrm{n}}}{\mathrm{m}\{(24 \mathrm{n}+\xi) /(24+\xi) \mathrm{n}\}^{\mathrm{n}} \xi^{\mathrm{n}-1}}
$$

With $\overline{\mathrm{u}}$ is the mean velocity flow and $\xi$ is the dimensionless geometrical parameter. We note that the dimensionless geometrical parameter $\xi$, is equal to 7.774 for an aspect ratio equal to $0.5[25]$.

\section{Grid Independency Test}

In order to check the sensitivity of the results to the mesh grid and to find the appropriate cell size, an unstructured grid with tetrahedral cells is implemented (Figure 2a), so five grid mesh, ranging from $437 \times 10^{3}$ to $1534 \times 10^{3}$, are tested in the TLCCM micromixer. In this examination, the power-law index and the generalized Reynolds number are taken 0.49 and 50 respectively. For this aim, the evolutions of the mixing index on different cross-sections along the channel for different grid mesh are presented, see Figure $2 b$. The difference between the grid mesh (1 534339 and 1323 058) don't exceed 1\% and subsequently, the choice is assigned to the grid with 1323058 cells in order to optimize the memory size. In addition, according to the sensitivity test results, the grid with 1323058 cells corresponds to a cell of $16 \mu \mathrm{m}$ in size, is sufficiently refined to find accurate results with a reduced calculation time.

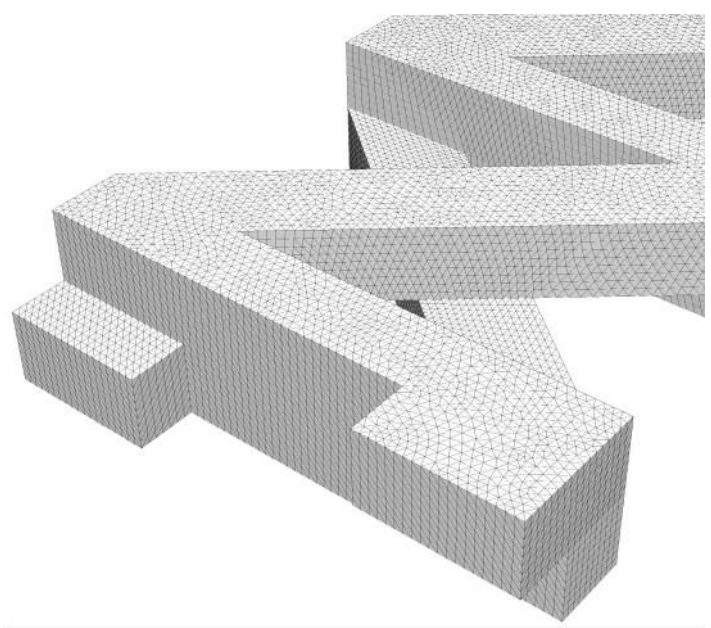

(a) 


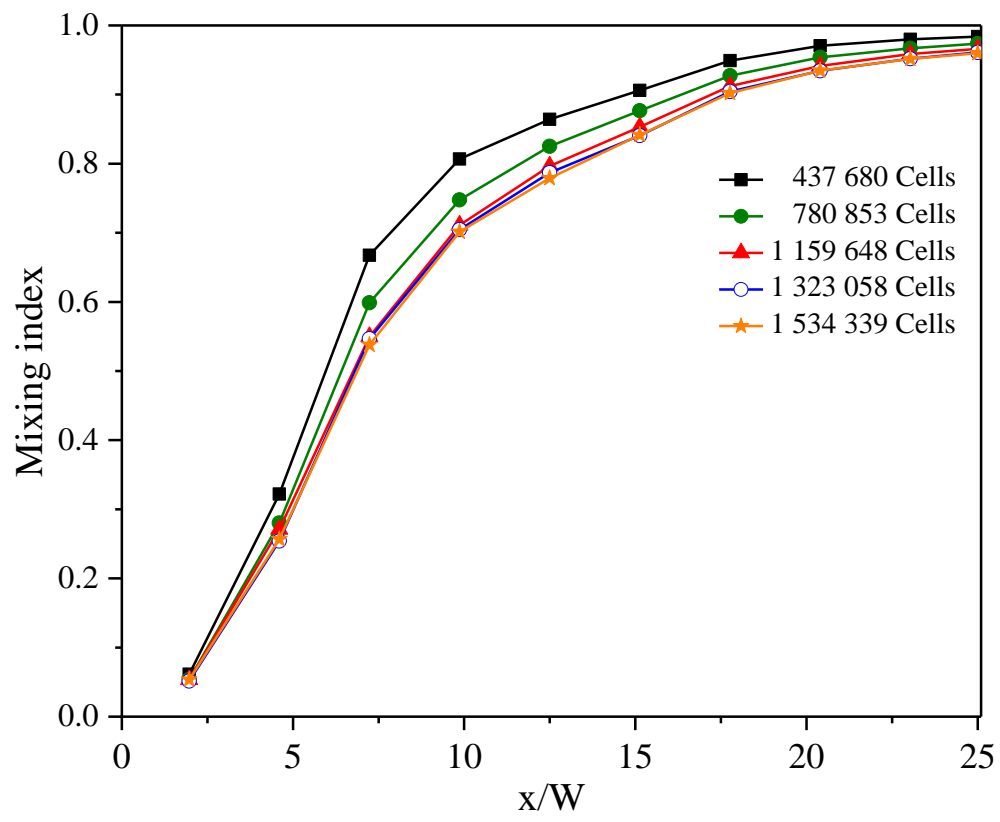

(b)

Figure 2. (a) Example of tetrahedral grid, (b) Mesh independency test for the evolution of mixing index $(\operatorname{Re}=50, \mathrm{n}=0.49)$.

\section{RESULTS AND DISCUSSION}

\section{Validation Tests}

Before using the CFD software, ANSYS FLUENT 16.0, validation tests must be carried out to ensure the reliability of the choice of calculation parameters. Such tests are done for both case studies; Newtonian and non-Newtonian fluids. A quantitative comparison was made with the existing results in the literature related to the mixing index and average apparent viscosity. They allow us to test and validate the Fluent calculation code by comparing our numerical results with those obtained in the literature in different geometries. For the Newtonian fluid, the mixing index calculated at the exit of the TLCCM micromixer for a Reynolds number between 0.2 and 120 was compared with the numerical and experimental results obtained by S. Hossain et al. [19], see Figure 3. The three evolutions are perfectly superimposed and the observed difference between the different results is barely remarkable. Where the maximum relative error with the numerical results is $6.16 \%$ while the maximum error with the experimental results is $4.59 \%$. The gap between the different evolutions remains in the range of the numerical method precision.

Concerning the non-Newtonian fluid case, the ratio of the average apparent viscosity of the CMC solutions to the viscosity of the deionized water (Newtonian fluid) and the mixing index were calculated and compared to that evaluated by Rei-Tang Tsai et al. [13] in the SCSM-90/34 micromixer, see Figure 4a. This comparison is done for a $500 \mathrm{ppm} \mathrm{CMC}$ solution where $\mathrm{n}=0.8229$.

The evolution of the average apparent viscosity ratio as a function of the mean velocity is presented on Figure $4 \mathrm{~b}$. The two evolutions are completely identical and the difference between the two curves is not at all observed, such as the maximum relative error 
is observed and only for the last velocity value which equal to $5.17 \%$. Figure $4 \mathrm{c}$ presents the evolution of the mixing index at the exit of the end of each unit of form $\mathrm{C}$ for mean velocity equal to $\overline{\mathrm{u}}=0.2187 \mathrm{~m} / \mathrm{s}$ in the SCSM-90/34 micromixer. Once again, the curves are in very good agreement with a maximum value of relative error equal to $12.47 \%$ and consequently, the relevance of the validation tests is shown.

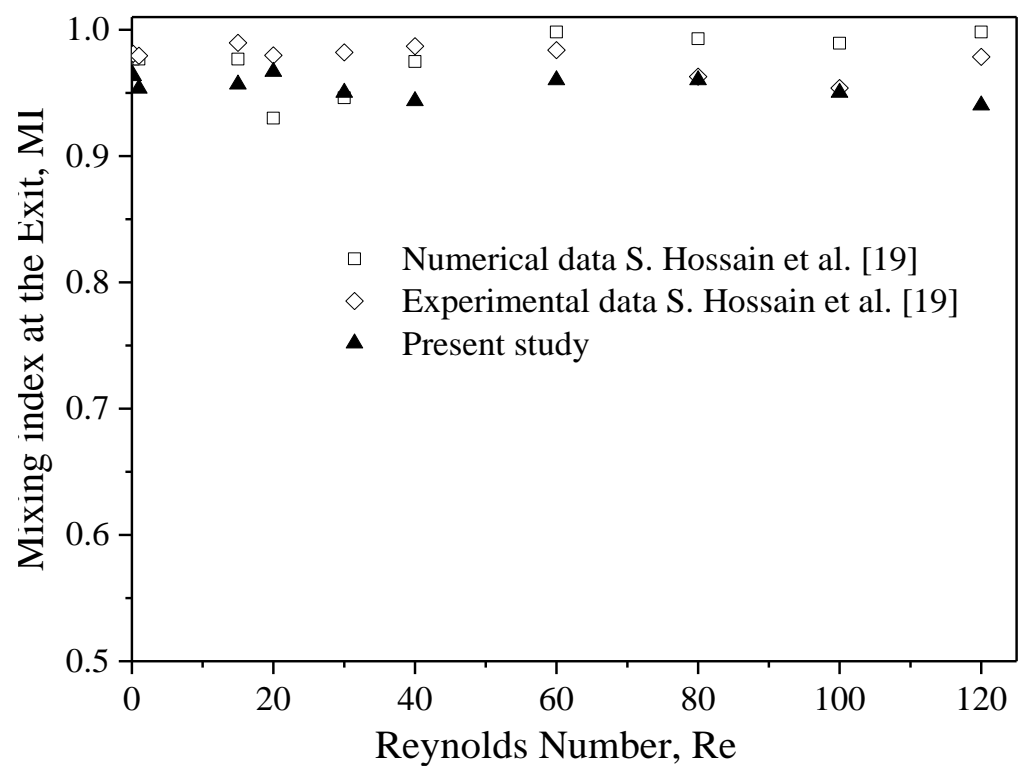

Figure 3. Evolutions of the mixing index as a function of Reynolds number for a Newtonian fluid for the current research and compared with S. Hossain et al [19].

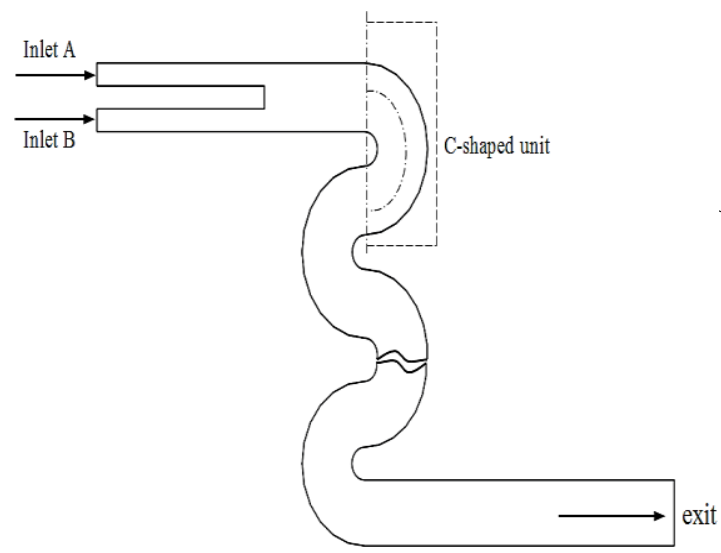

(a)

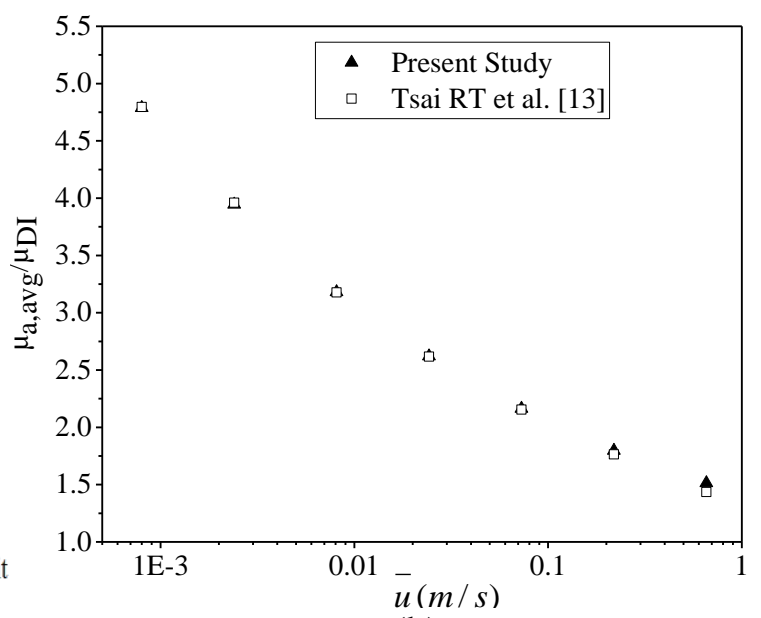

(b) 


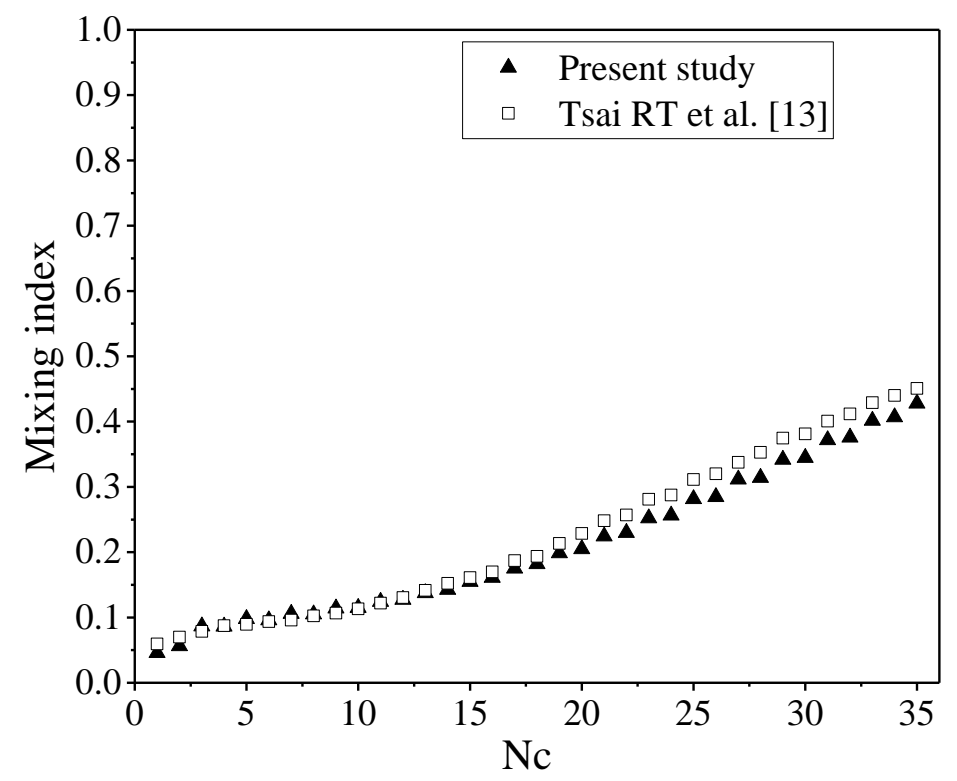

(c)

Figure 4. Comparison of the results for non-Newtonian fluid:

(a) Micromixer geometry [13], (b) Average apparent viscosity ratio as compared with [13], (c) Mixing index at the exit of each C-shaped unit compared with [13].

\section{Investigation of the Results}

\section{Dynamic behaviour}

In the dynamic systems theory, the flow structure is complex if there is at least one unstable element somewhere in the phase's space. These unstable elements in the phase's space are hyperbolic points, unlike elliptic points [26]. Hyperbolic and elliptical points are schematized in Figure 5. The region formed by an elliptical point and its close vicinity represents an obstacle to an effective mixing. As for the hyperbolic point, we associate two local behaviors: one stable Ws and the other unstable Wi, ("s" for stable and "i" for unstable) [10]. The stable directions, Ws, and unstable, $\mathrm{Wi}$, respectively correspond to the physical directions of compression and stretching, see Figure 6.
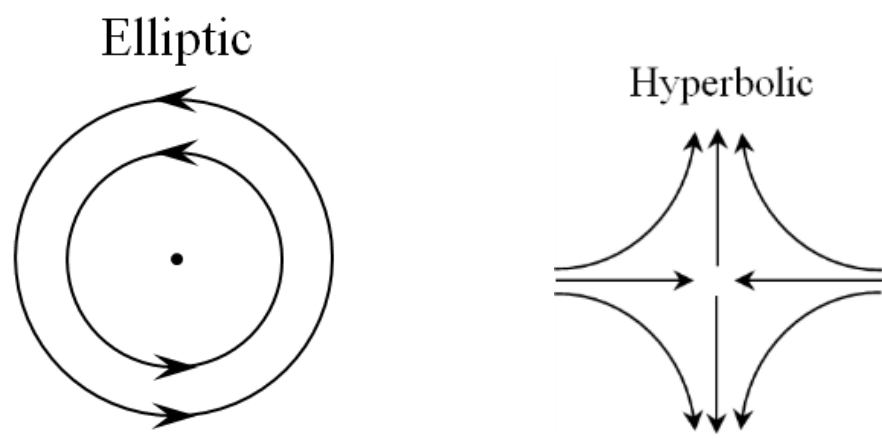

Figure 5. Elliptic and Hyperbolic Points [26]. 


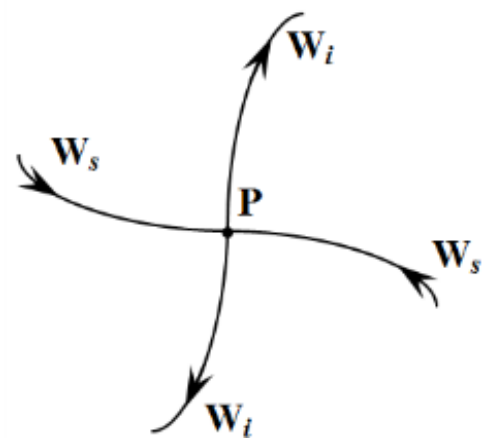

Figure 6. Stable behavior Ws and unstable behavior Wi for hyperbolic points [26].

This behaviour is a strong sign of the appearance of chaotic advection in the flow [9]. The intersection of the stable and unstable elements in the three planes even at low Reynolds numbers is clearly visualized in our micromixer, see Figure 7, where the velocity vectors are plotted on the $y$-z planes at the nodes of the cross-sections in the mixing channel for $\mathrm{Re}=0.1$ and $n=1$, this figure visualizes the flow pattern with a saddle-shaped [17], which is an important factor in the formation of chaotic advection. The saddle-shaped consists as an hyperbolic which split into four zones of fluid streams, with two parabolic segments (up and down) and two helical (left and right) flow patterns, thus result from the two-layer crossing while the fluids are driven from the bottom-layer channel to the top-layer channel and the reverse, it causes the stretching and compression of the fluids. In all planes $\left(\mathrm{P}_{1}-\mathrm{P}_{9}\right)$, the fluid interface is narrowed along diagonal line, while that of the other diagonal line is stretched, as shown by the direction of the arrows of velocity vectors, this phenomenon compression and stretching is one of the key mechanisms for expanding the interfacial layer of fluids, which improves mixing. Therefore, the strong flow structures at the nodes of the crossing channels gave excellent mixing performance for the present micromixer. This figure also reveals that the distribution of velocity vectors in all crossing planes remains almost identical and stable.

\section{Plane $\mathrm{P}_{1}$}

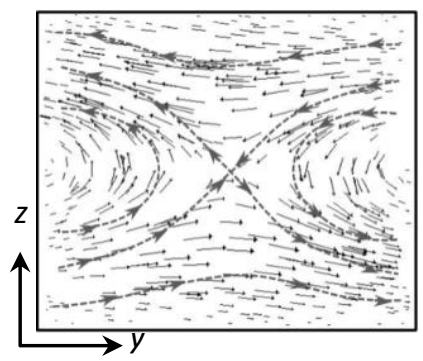

Plane $\mathrm{P}_{5}$

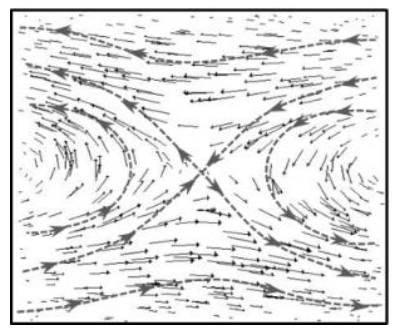

Plane $\mathrm{P}_{9}$

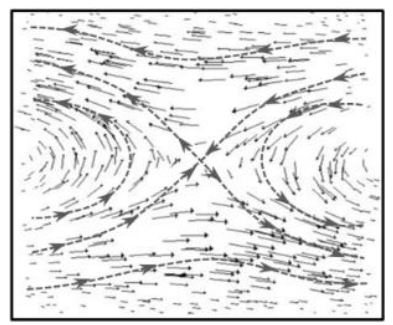

Figure 7. Velocity vectors on $y-z$ planes at the nodes cross-sections for $\operatorname{Re}=0.1$ and $n=1$.

Figures 8 and 9 show the secondary flows in planes 5, AA and BB for two power-law indices 0.73 and 1 and two generalized Reynolds numbers 0.1 and 50. The contour of the velocity vectors describes both the crossing of the flows at plane 5 arriving from the top and the bottom of the channel and the formation of the two great vortices in the cross-sections 
$\mathrm{AA}$ and $\mathrm{BB}$, even at low power-law indices and low generalized Reynolds numbers (creeping flows). The crossing flows occurring inflows resemble the collision of two jets fluids. Fluid jets are very effective means to improve the mixing in a fast way even at low Reynolds numbers [17]. By increasing the Reynolds number, the vortices formed at the cross-sections are more intense and have a different vortex structure [19]. The crossing of the streamlines and the descending and ascending flows are prevailing by the tangential and radial velocity profiles as shown on the Figures 10 and 11 respectively. In addition, the maximum of the velocity is located close to the wall for all cases of studies considered. This allows stagnant fluids near walls to move to the core of the cross-section. The axial velocity profiles show that the flow is decelerated in the center of the pipe and is accelerated near the side walls. This reveals that the secondary flow is generated and it is more or less important depending on the values of the Reynolds number and the power-law index, see Figure 12.

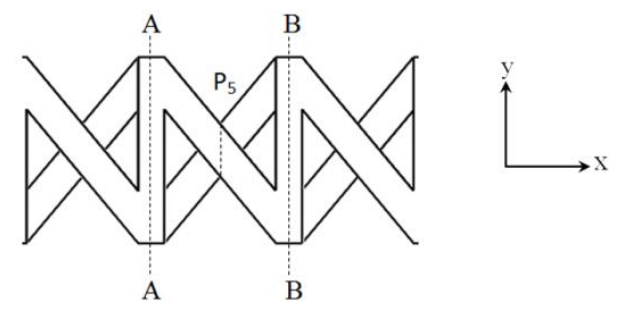

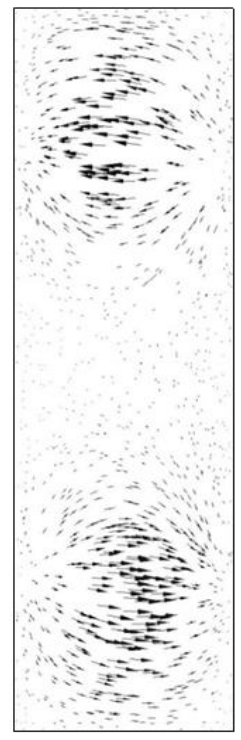

A-A

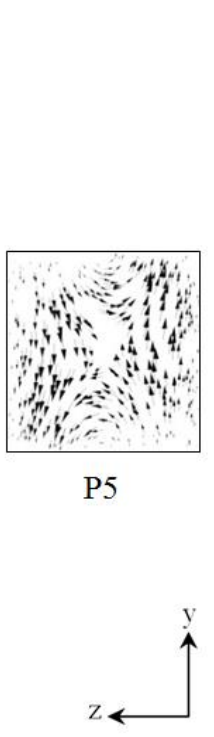

(a)

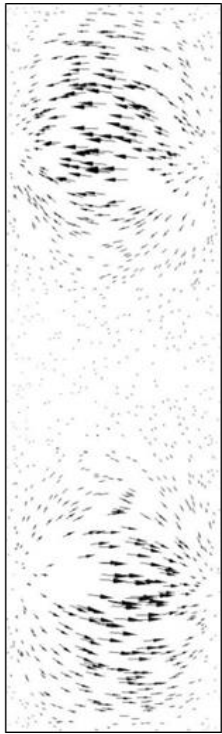

B-B

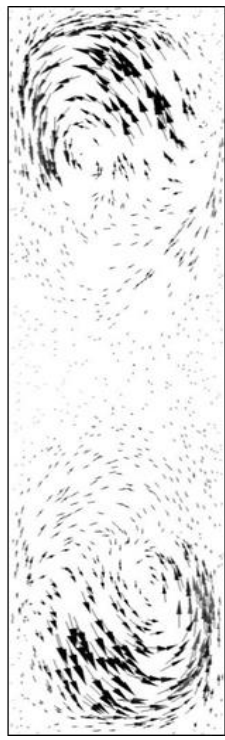

A-A

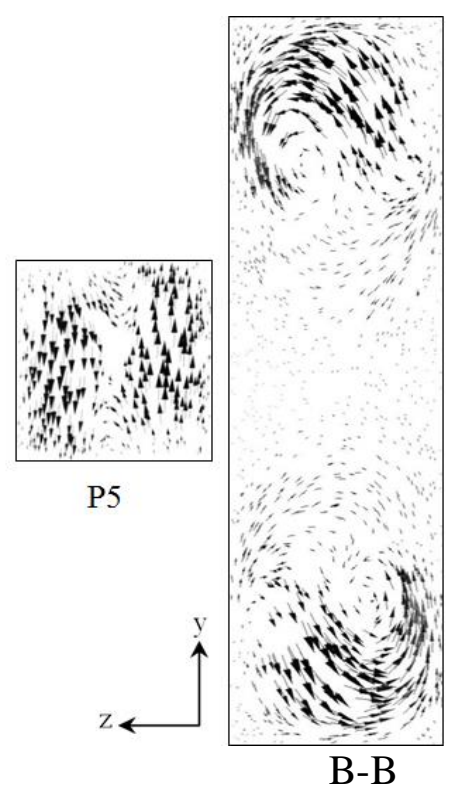

(b)

Figure 8. Velocity vector plots on $y-z$ planes $n=1$, (a) $\operatorname{Re}=0.1$, (b) $\operatorname{Re}=50$. 

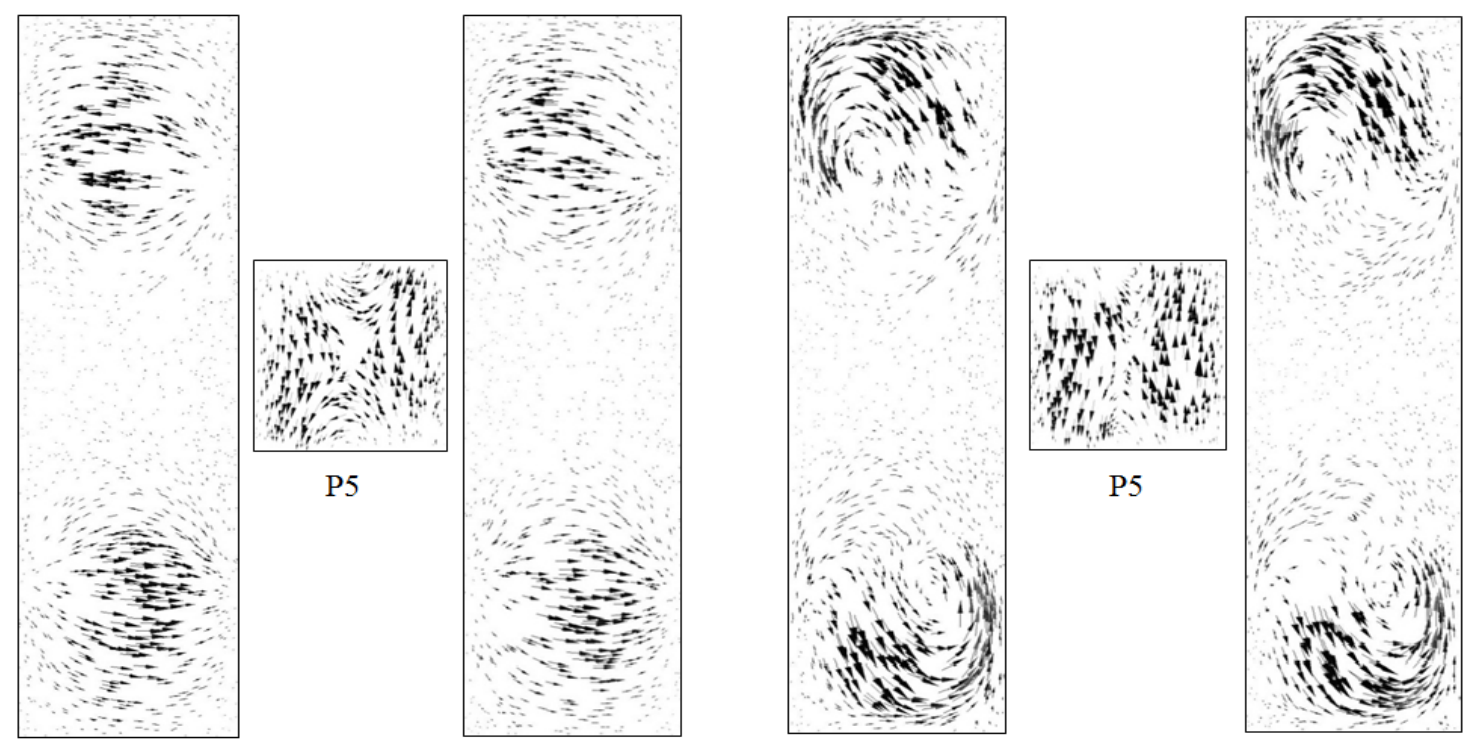

(a)

(b)

Figure 9. Velocity vector plots on $y-z$ planes, $n=0.73$, (a) $\operatorname{Re}=0.1$, (b) $\operatorname{Re}=50$.

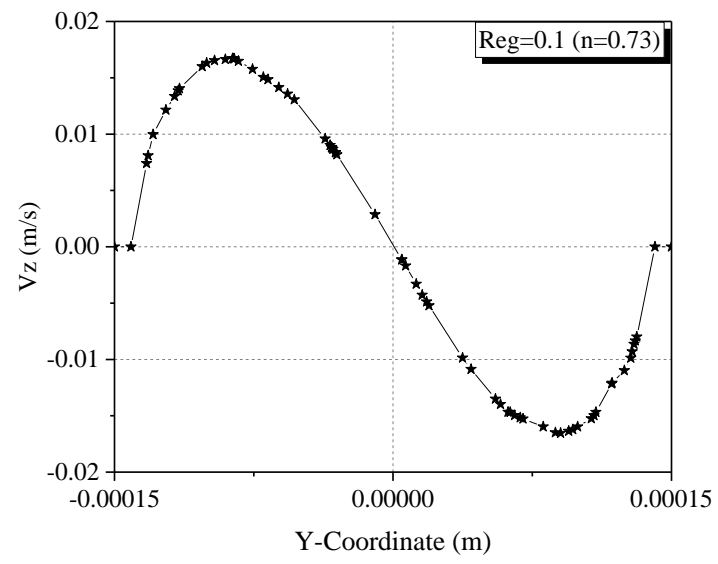

$\left(\mathrm{a}_{1}\right)$

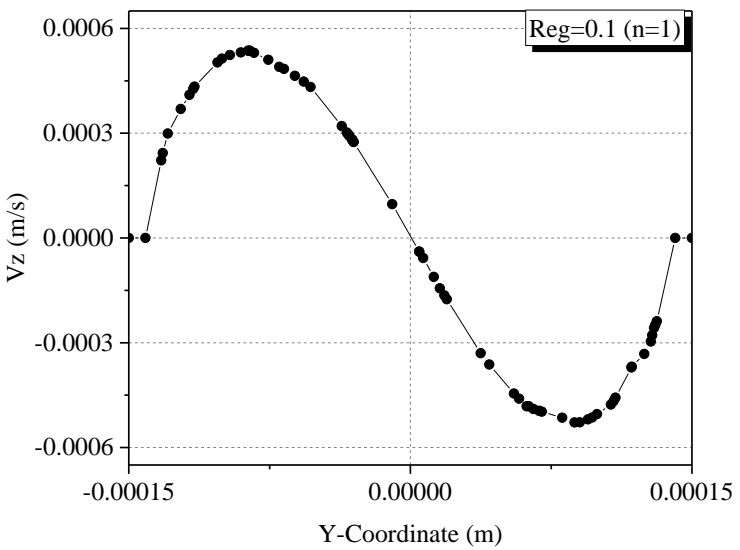

$\left(a_{2}\right)$ 


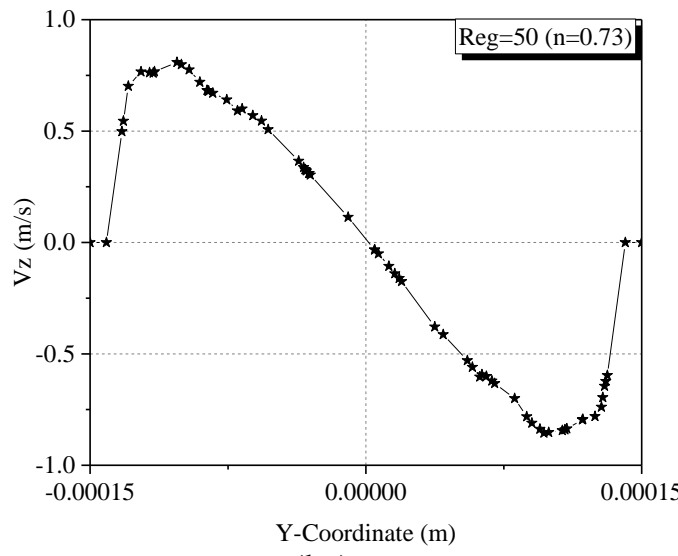

$\left(b_{1}\right)$

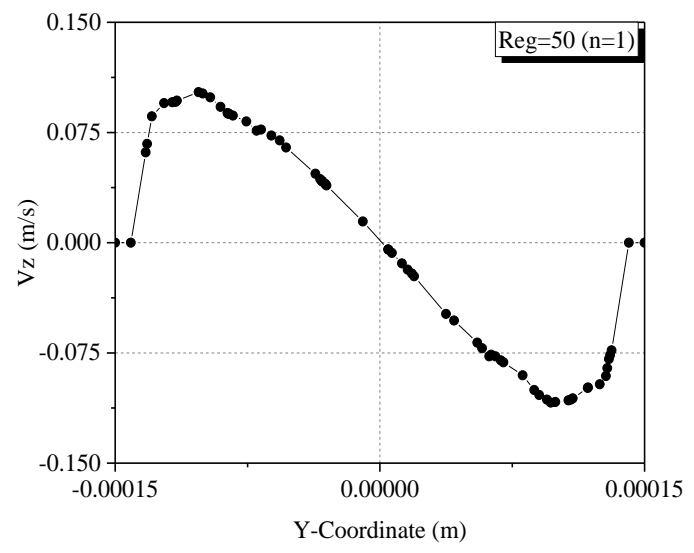

$\left(b_{2}\right)$

Figure 10. Tangential velocity profiles at $\mathrm{P}_{5}$ plane for $\mathrm{n}=0.73$ and $1,\left(\mathrm{a}_{1}, \mathrm{a}_{2}\right) \operatorname{Re}_{\mathrm{g}}=0.1$, $\left(b_{1}, b_{2}\right) R_{e}=50$.

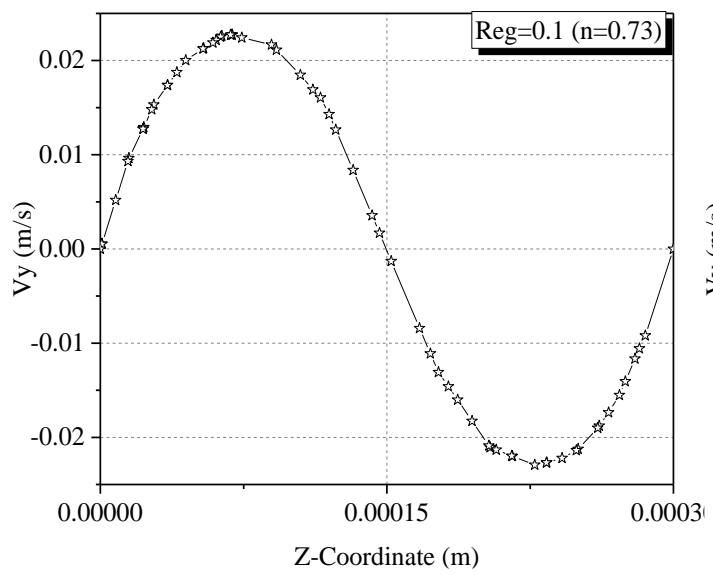

$\left(\mathrm{a}_{1}\right)$

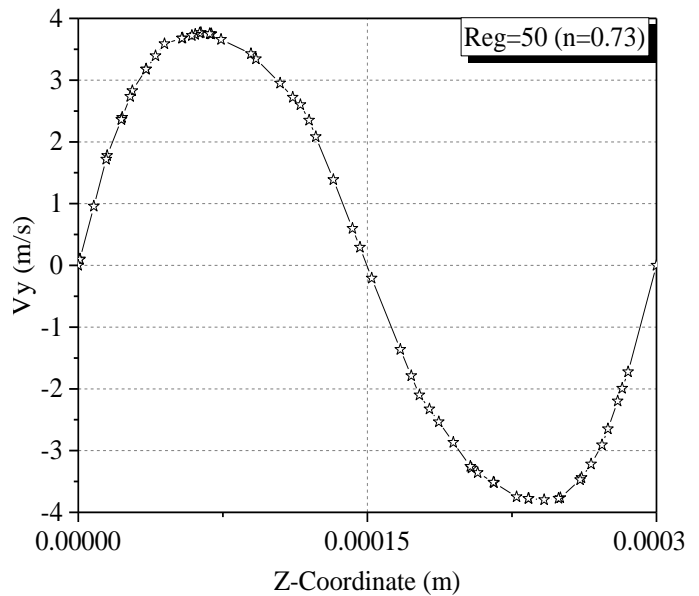

$\left(b_{1}\right)$

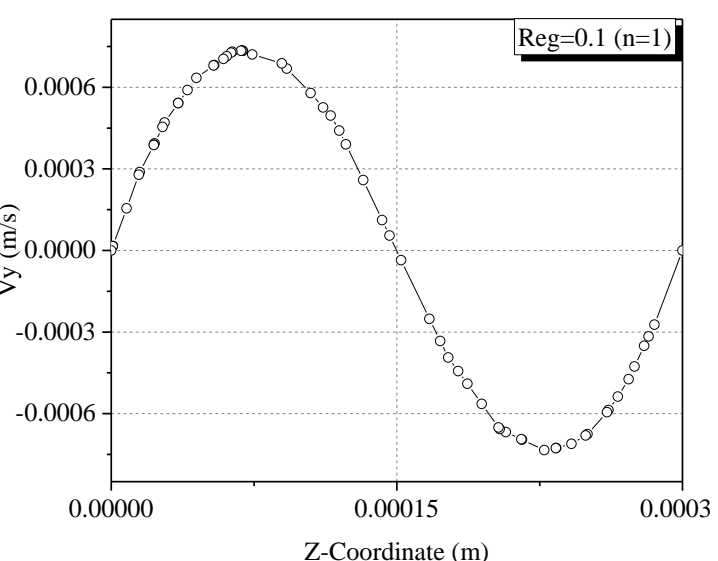

$\left(\mathrm{a}_{2}\right)$

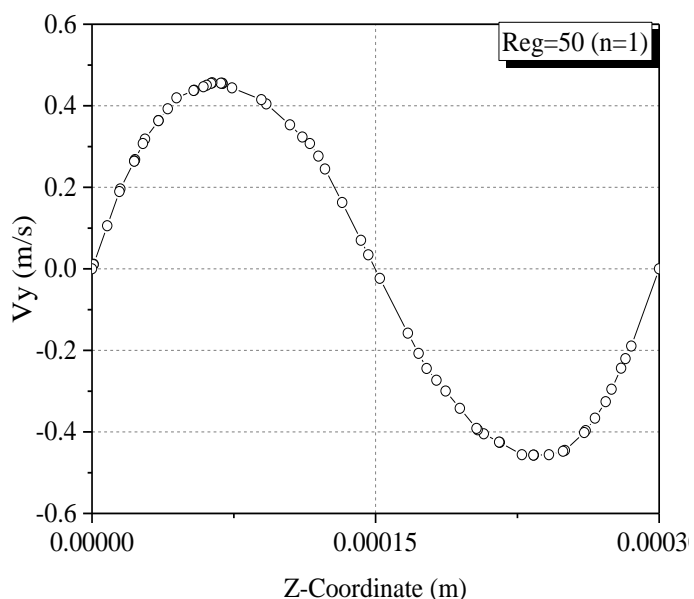

$\left(b_{2}\right)$

Figure 11. Radial velocity profiles at $\mathrm{P}_{5}$ plane for $\mathrm{n}=0.73$ and 1 , $\left(a_{1}, a_{2}\right) \operatorname{Re}_{g}=0.1,\left(b_{1}, b_{2}\right) \operatorname{Re}_{g}=50$. 


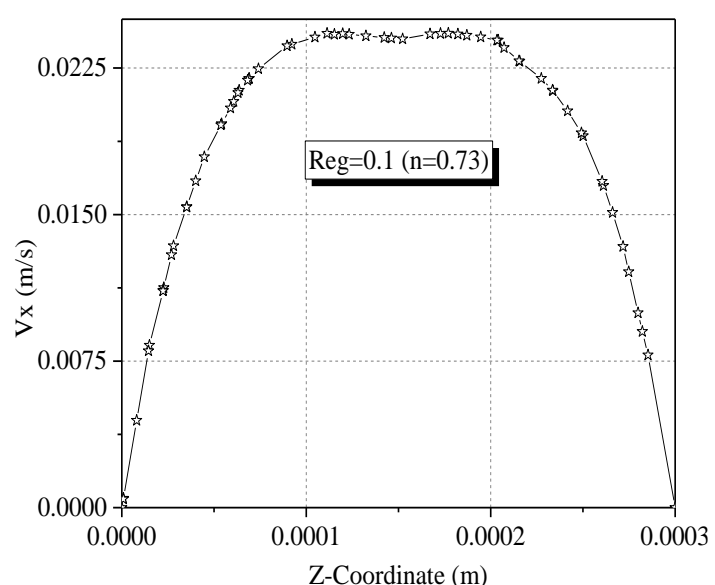

$\left(\mathrm{a}_{1}\right)$

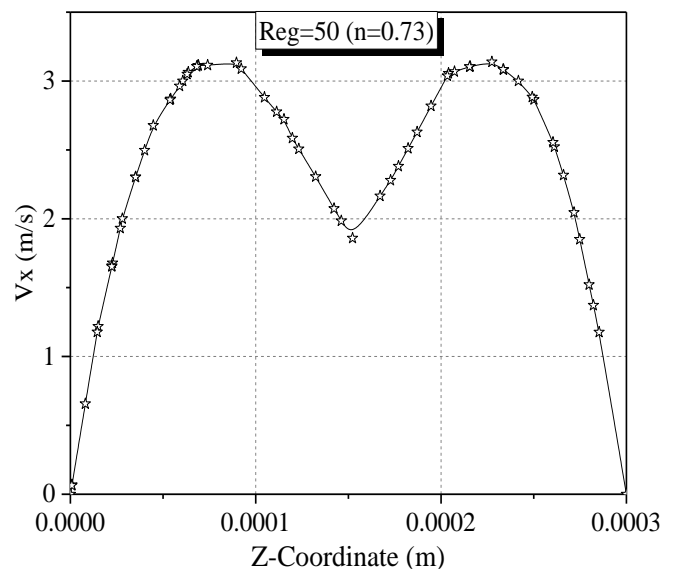

$\left(b_{1}\right)$

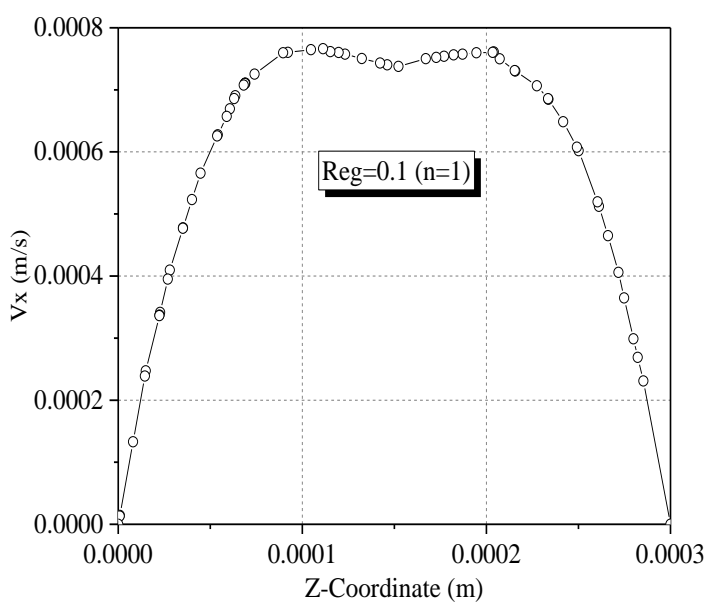

$\left(\mathrm{a}_{2}\right)$

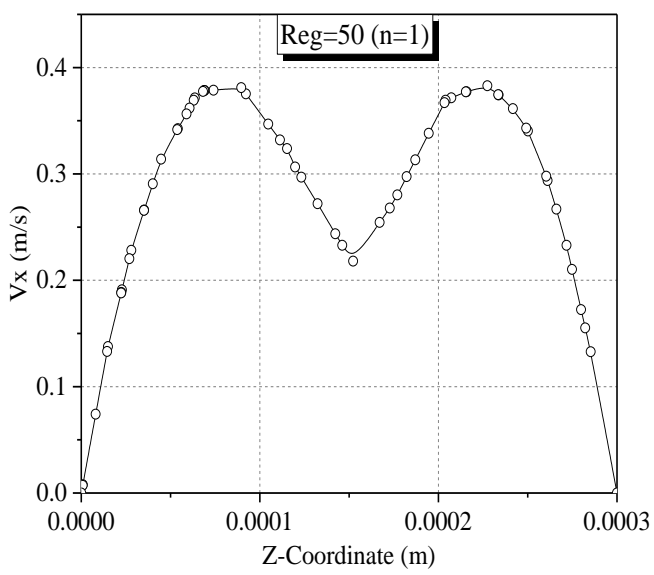

$\left(b_{2}\right)$

Figure 12. Axial velocity profiles at $\mathrm{P}_{5}$ plane for $\mathrm{n}=0.73$ and 1 ,

$$
\left(a_{1}, a_{2}\right) \operatorname{Re}_{g}=0.1,\left(b_{1}, b_{2}\right) \operatorname{Re}_{g}=50 \text {. }
$$

The intensity is defined by the following equation:

$$
I=\frac{\sqrt{V_{y}^{2}+V_{z}^{2}}}{|V|}
$$

Equation (11) estimated the degree of agitation of the flows in a cross-section. It represents a local criterion for characterizing how much the axial flow is decreased, see Figure 13. This parameter shows that the secondary flow represents more than 80 percent of the total flow and this is true for all values of the Reynolds number and the power-index with however a slight accentuation of the secondary flow for small values of $\mathrm{n}$ so for great values of the apparent viscosity. The increase of secondary flows for large apparent viscosity values is explained by the fact that the viscosity decelerates the flow by the friction. Thus, the viscosity allows the fluid particles to move in bloc motion. In low viscosity flows (perfect fluid for example), the fluid particles move individually and freely. 


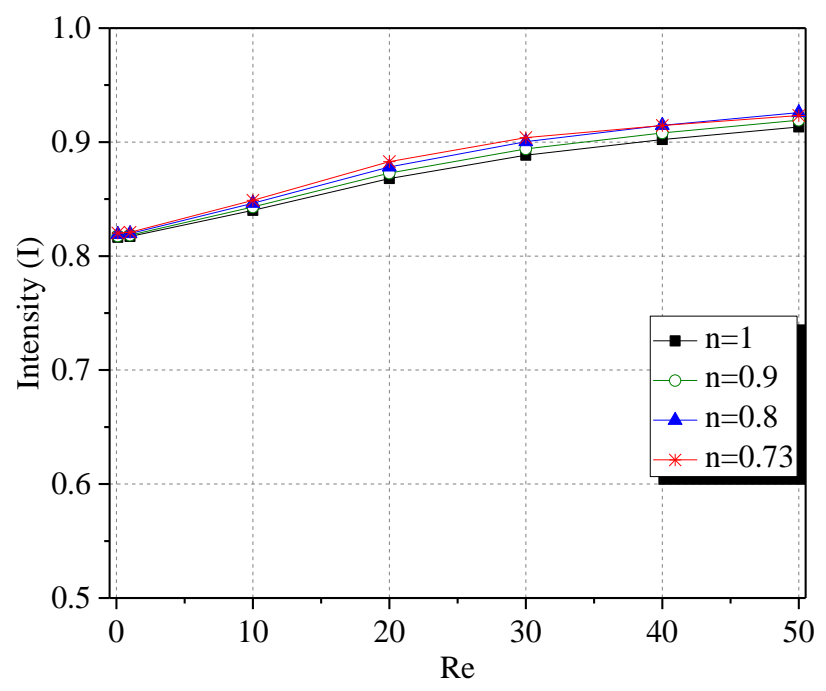

Figure 13. Evolutions of the intensity I in the $\mathrm{P}_{5}$ plane with generalized Reynolds number for different power-law index $(n=0.73$ to 1$)$.

\section{The mixing performances of the TLCCM micromixer}

Figure 14a shows the evolutions of the mixing index with the generalized Reynolds numbers which vary from 0.1 to 50 at the exit cross-section of the TLCCM micromixer for different values of the power-law index ranges from 0.73 to 1 . It can be seen that high mixing quality is reached even at very low generalized Reynolds number (from 0.1 ) of which the mixing index exceeds $96 \%$ in the adopted range of generalized Reynolds number and for any values of the power-law index less than 1. Figure 14(b-h) illustrated the local evolutions of the mixing index along the duct for all values of the generalized numbers and power-law indices. Remarkably, the curves which correspond to each $\mathrm{n}$ are distant from each other mainly for 0.1 and 1 values of $\operatorname{Re}_{\mathrm{g}}$ where the mixing is better for the large values of $\mathrm{n}$. By increasing the Reynolds number, the curves get closer because the motion of the particles by advection is more highlighted and intense, so the difference between the curves is scarcely measured and the effect of $\mathrm{n}$ is canceled and Figure 15 illustrates the mass fraction contours in several crosssections for two generalized Reynolds numbers ( 0.1 and 1$)$ according to a power-law index less than 1. By lengthening the pipe, the mixing arises after a short distance traversed by the fluid in the channel. So, these contours revealed the efficient mixing even at creeping flow as soon as we are not really far from the entrance. In addition, the mixing is proportional to the power-law index as mentioned above in Figures 14 and 15. This is explained by the fact that the increase of the power-law index induces the decreases of the apparent viscosity and subsequently the agitation of the fluid becomes easier, see Figure 16. The diminution of the viscosity (augmentation of $\mathrm{n}$ ) introduces in the flow low shear rates, see Figure 17. Low shear rates increase mixing in quality and speed.

However, the apparent viscosity for a Newtonian fluid maintains almost constant values whatever the Reynolds number (Figure 18). 

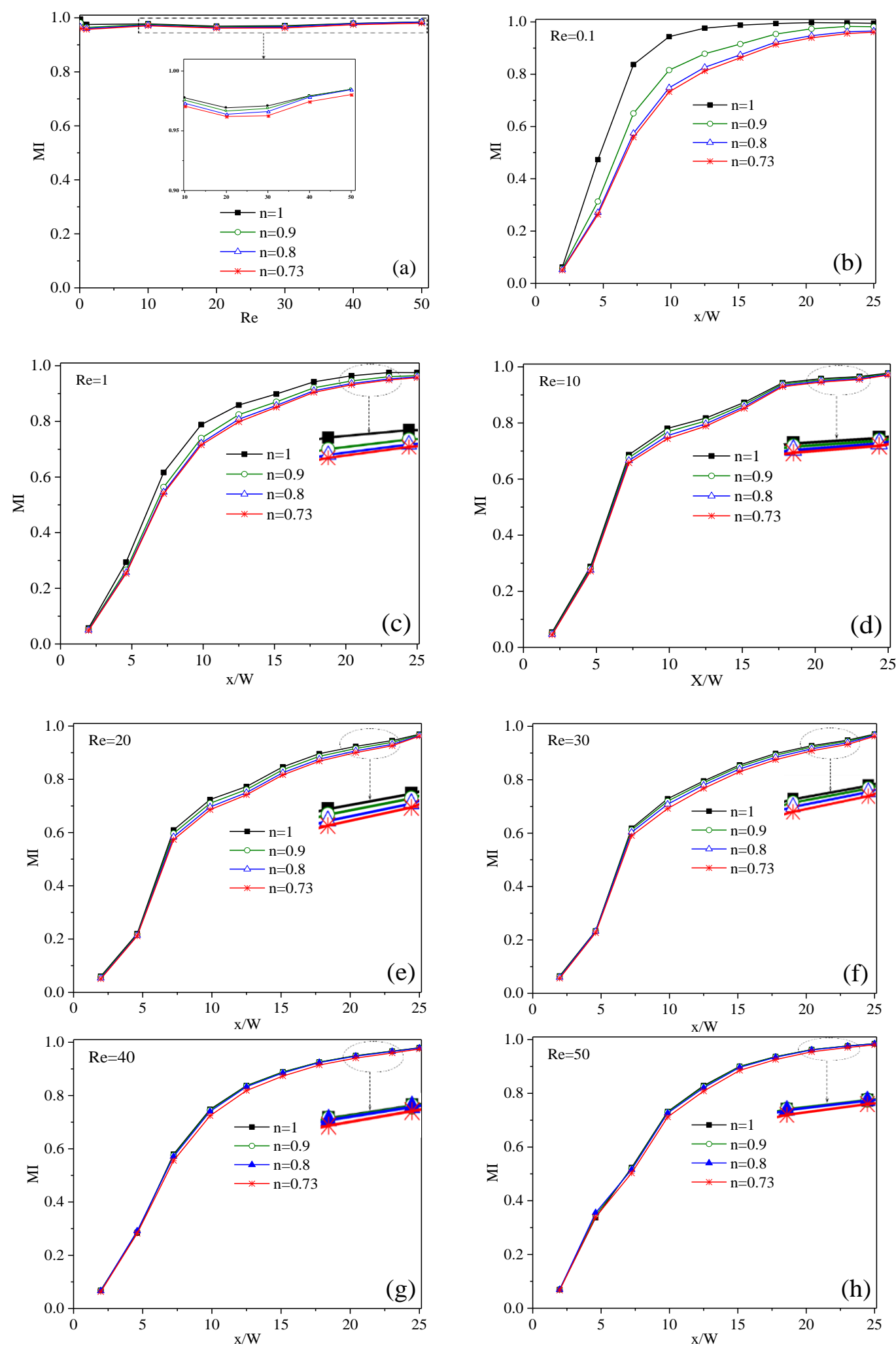

Figure 14. (a) Variation of mixing index at the exit. (b) to (h) Evolutions of mixing index along the mixing channel for different power-law indices and various Reynolds numbers. 


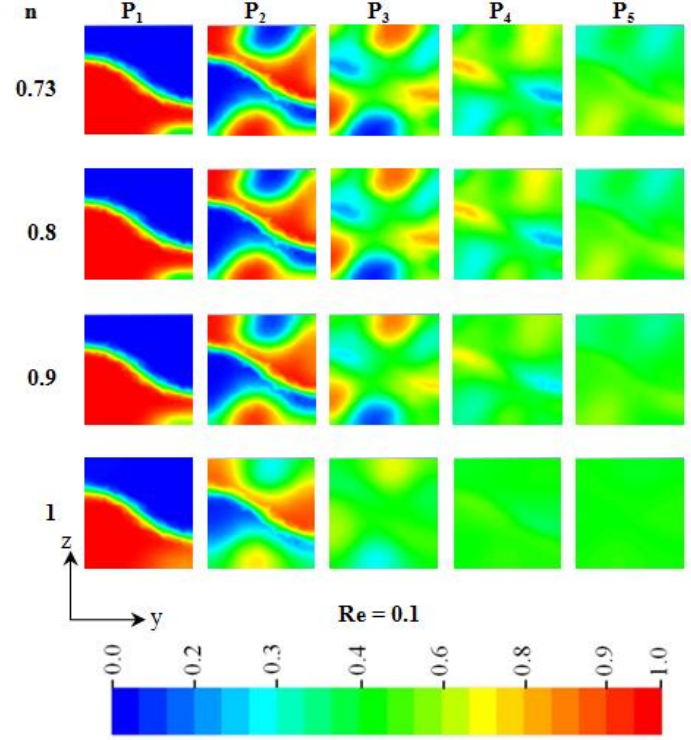

(a)

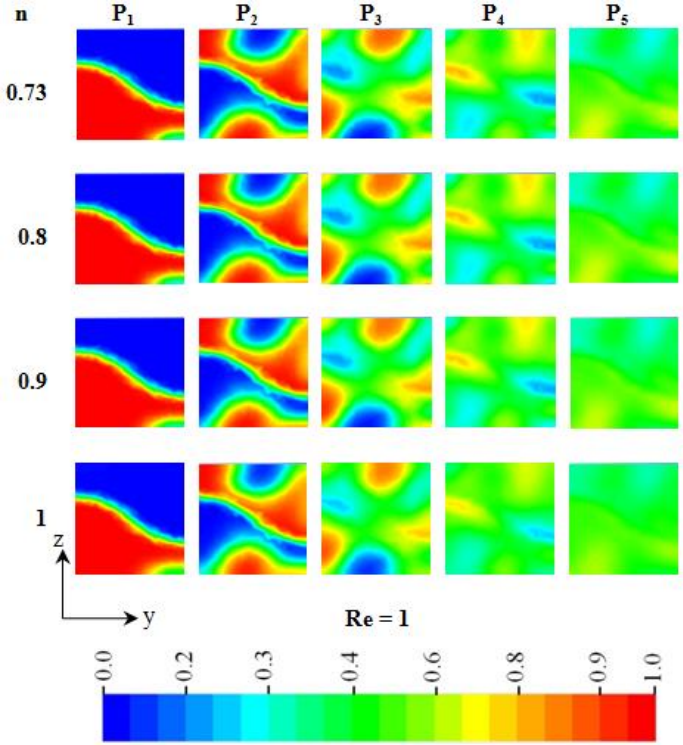

(b)

Figure 15. Mass fraction contours at different cross-sections for various power-law indices, (a) $\operatorname{Re}=0.1$, (b) $\operatorname{Re}=1$.

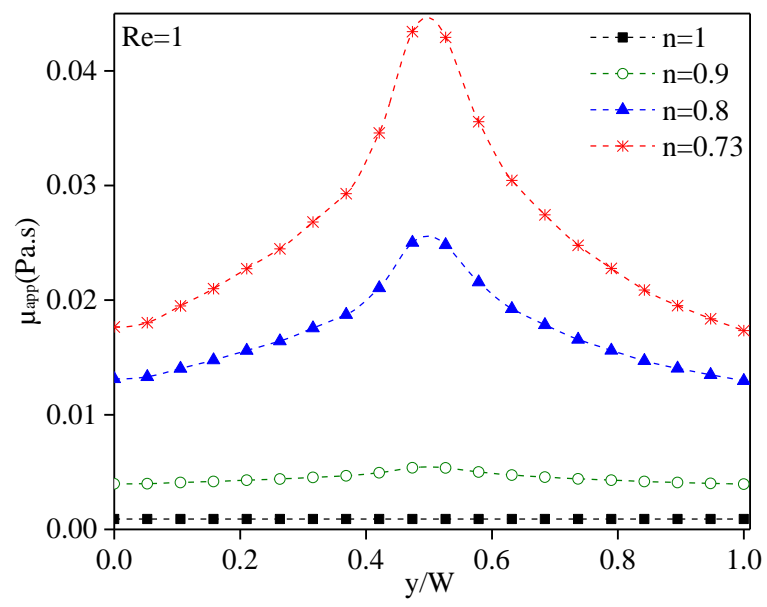

Figure 16. Apparent viscosity profiles on the line $\mathrm{z}=0$ at the exit plane for different powerlaw indices. 


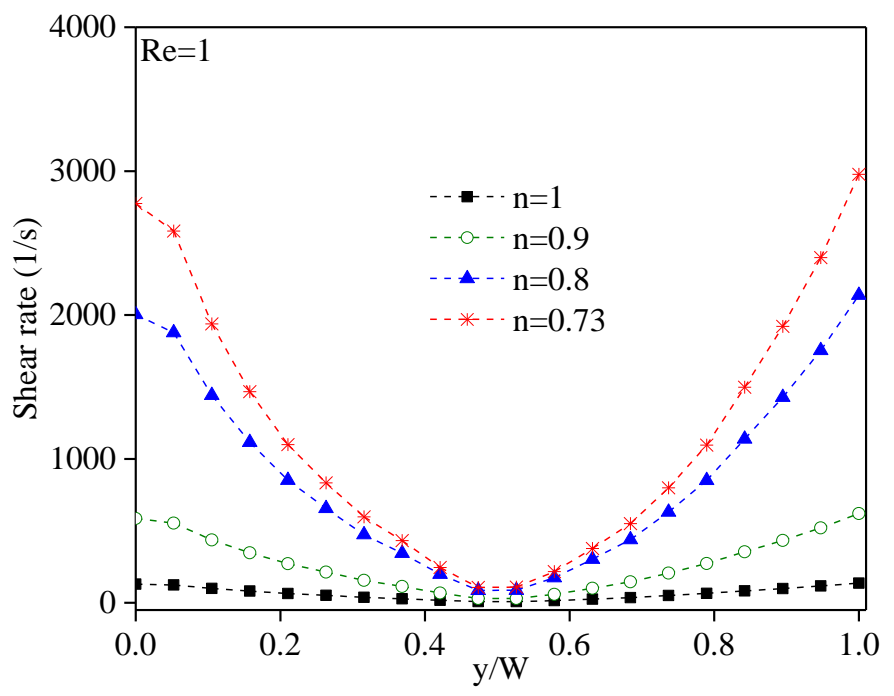

Figure 17. Shear rate profiles on the line $\mathrm{z}=0$ at the exit plane for different power-law indices.
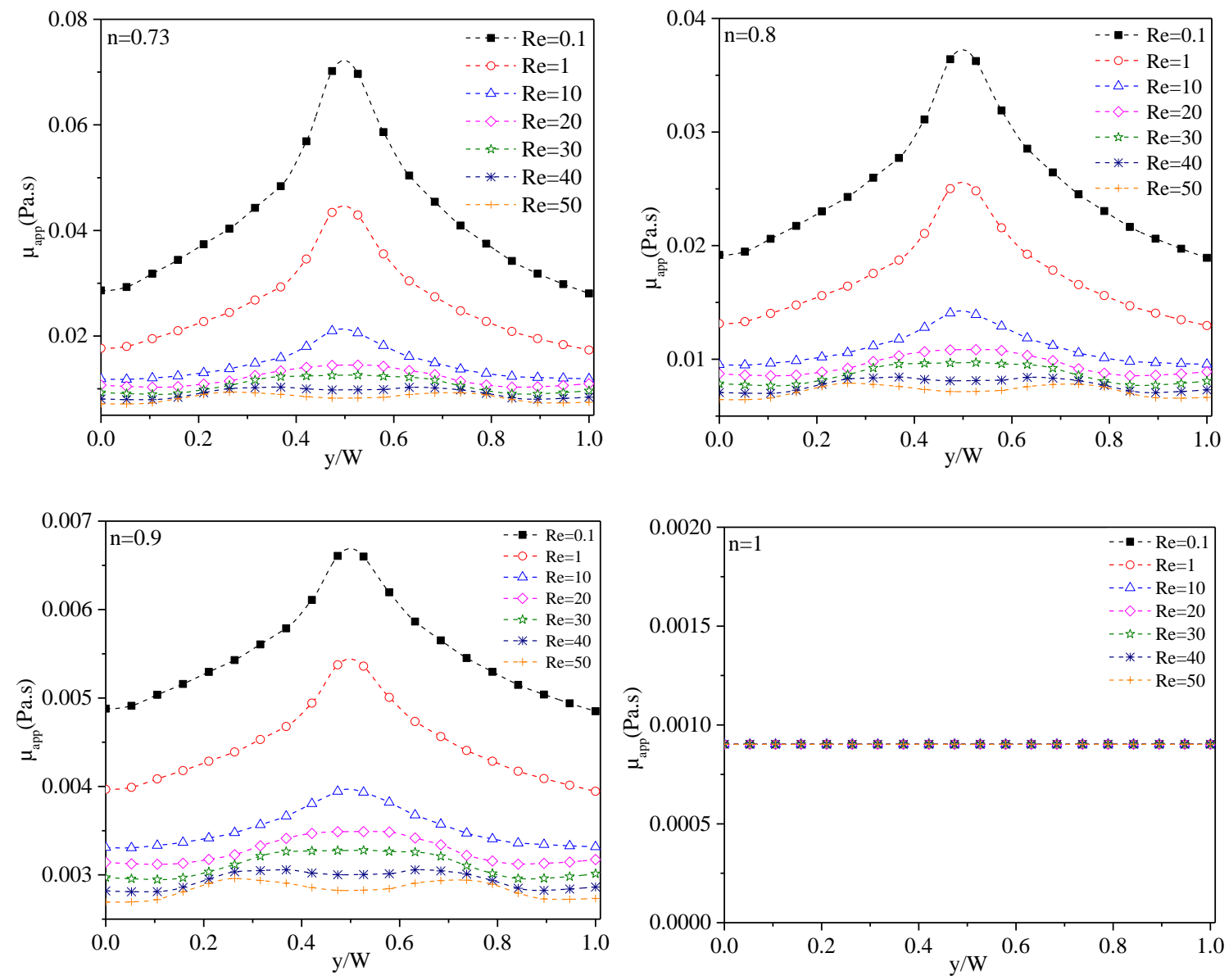

Figure 18. Apparent viscosity profiles on the line $\mathrm{z}=0$ at the exit plane for different Reynolds numbers. 
The mixing performance of other typical micromixers contains shear-thinning fluids involving T-shaped and serpentine microchannels (Afzal A et al. [12]), micromixer with Cshaped units (Tsai RT et al. [13]), micromixer with curved units (Islami SB et al. [15]), micromixer with Curved and Grooved units (Islami SB et al. [16]), E-shape split-andrecombine micomixer ( $\mathrm{He} \mathrm{M}$ et al. [30]), and the micromixers contains Newtonian fluids, involving two layers with $\mathrm{OH}$-shaped units (Hossain S et al. [28]), and recently two layers OX-shaped units (Raza W et al. [29]). A quantitative comparison has been carried out to compare the mixing index of our micromixer with those of recent studies on various micromixers as shown in Table 2. It's indicated that the mixing index can be more than $90 \%$ under certain Reynolds number for these micromixers. However, the present micromixer have a greater values of mixing index which varied from 0.96 to 0.99 at very low and moderate Reynolds numbers ( $R e \geq 1$ ), which clearly shows the superiority of our micromixer.

Table 2. Comparison of the TLCCM for the mixing index with recent micromixers.

\begin{tabular}{|c|c|c|c|c|}
\hline Micromixers & $\mathbf{n}$ & $\mathbf{R e}$ & MI & Authors \\
\hline $\begin{array}{l}\text { T-shaped and } \\
\text { serpentine }\end{array}$ & $(\mathrm{n}<1)$ & $0.05-100$ & $0.37-0.98$ & Afzal A et al. [12] \\
\hline C-shaped units & $0.82-0.95$ & $0.021-57.76$ & $0.14-0.94$ & Tsai RT et al. [13] \\
\hline Curved units & $0.49-1$ & $0.1-300$ & $0.07-0.99$ & Islami SB et al. [15] \\
\hline $\begin{array}{l}\text { Curved and Grooved } \\
\text { units }\end{array}$ & $0.49-1$ & $0.1-300$ & $0.05-0.97$ & Islami SB et al. [16] \\
\hline $\begin{array}{l}\text { E-shaped split-and- } \\
\text { recombine }\end{array}$ & $0.69-1$ & $0.05-100$ & $0.95-0.99$ & He $\mathrm{M}$ et al. [30] \\
\hline $\begin{array}{l}\text { Two layers with OH- } \\
\text { shaped units }\end{array}$ & 1 & $0.1-120$ & $0.61-0.88$ & Hossain S et al. [28] \\
\hline $\begin{array}{l}\text { Two layers with OX- } \\
\text { shaped units }\end{array}$ & 1 & $0.1-120$ & $0.87-0.99$ & Raza W et al. [29] \\
\hline TLCCM & $0.73-1$ & $0.1-50$ & $0.96-0.99$ & Present study \\
\hline
\end{tabular}

A high mixing performance of the micromixer is usually accompanied by a highpressure drop. The pressure difference between the inlet and the outlet cross-sections of the channels was calculated and presented according to the Reynolds number for the different values of the power-law index as presented in Figure 19. By increasing the Reynolds number, the pressure drop increases with Reynolds number and decreases with the increase of the power-law index (the decrease of the apparent viscosity) because it is proportional to the apparent viscosity. The latter generates friction on the one hand between the fluid layers and on the other hand between the fluid and the wall. The contribution of the apparent viscosity to the increase in friction is shown in Figure 20. 


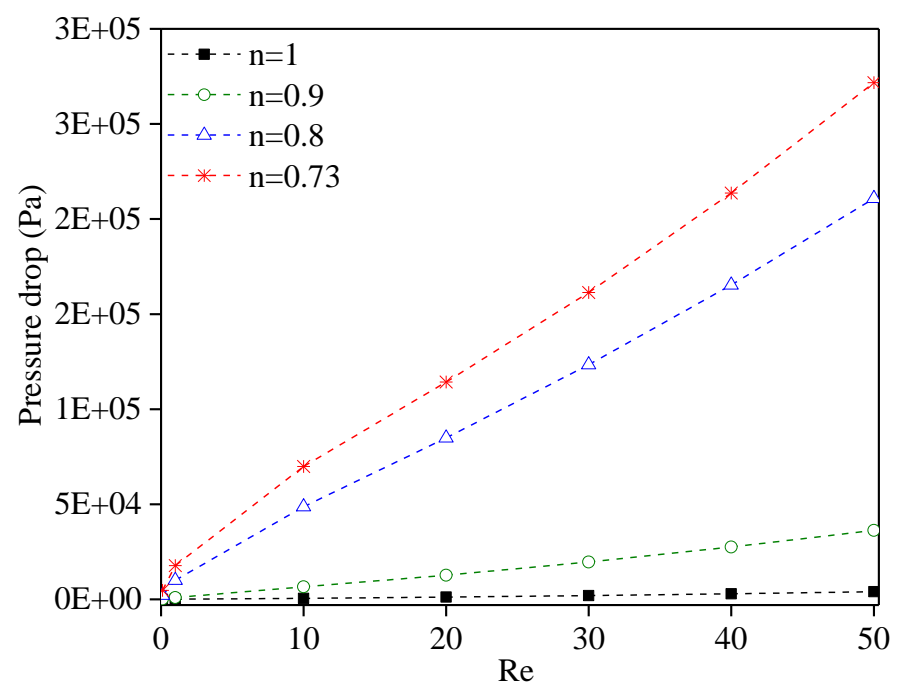

Figure 19. Variation of Pressure drops with the Reynolds numbers for different power-law indices.

To examine the compromise between the pressure drops and the kinetic energy, we are interested to calculate the friction factor $\mathrm{f}$ which is defined by the following equation:

$$
\mathrm{f}=\frac{\Delta \mathrm{P}}{\frac{1}{2} \rho \overline{\mathrm{u}}^{2}} \frac{\mathrm{D}_{\mathrm{h}}}{\mathrm{L}}
$$

Where $\Delta \mathrm{P}$ is the pressure difference between the inlet and outlet sections, $\mathrm{D}_{\mathrm{h}}$ is the hydraulic diameter of the micromixer and $\overline{\mathrm{u}}$ is the average flow velocity. It has a decreasing tendency according to the Reynolds number, see Figure 20.

Figure 20 shows the evolutions of the friction factor with Reynolds number for different power-law indices. We observe that the friction factor decreases with increasing Reynolds number and their values are almost identical for all the fluids considered.

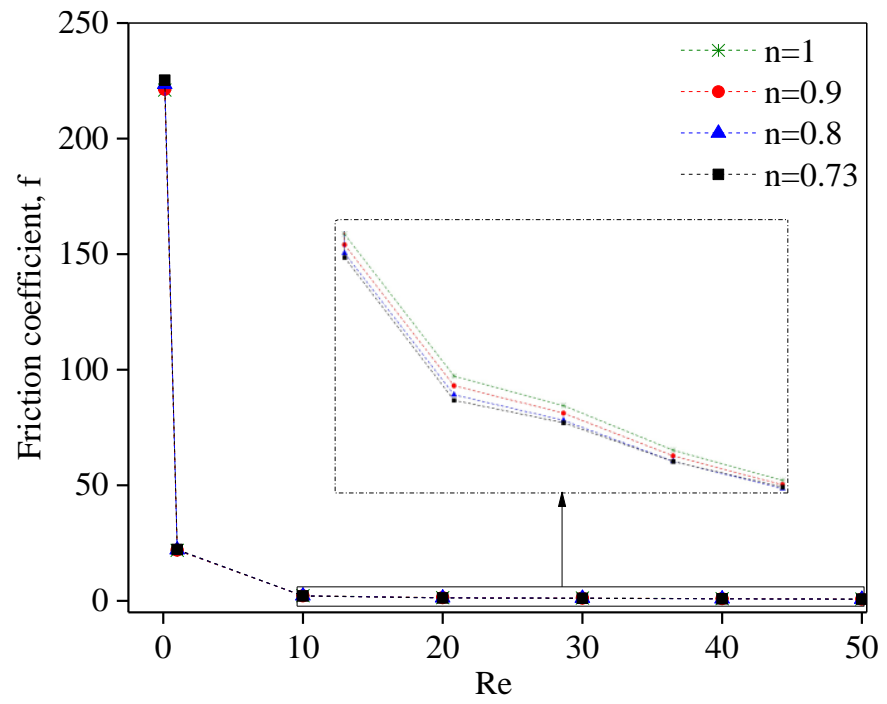

Figure 20. Variation of friction factor with the Reynolds numbers for different power-law indices 
The Poiseuille number is too used for the characterization of the pressure losses. It is equal to the product between the local friction factor $\mathrm{f}$ and the Reynolds number according to the Equation. (13):

$$
\text { Po }=\text { f. Re }
$$

Figure 21 presents the evolution of the Poiseuille number with the Reynolds number for different values of the power-law indices $n$. This curve revealed that the Poiseuille number evolves continually/inversely with the power-law index/viscosity apparent, respectively. We can also observe that the evolution of the Poiseuille number starts with low values of the Reynolds number ranging between 0.1 and 10 and then rapidly increasing. The Poiseuille number calculated in this geometry does not exceed 40 whereas the Poiseuille number according to a straight tube of the square section is equal to 60. Therefore, the pressure losses in the TLCCM geometry are not in fact considerable.

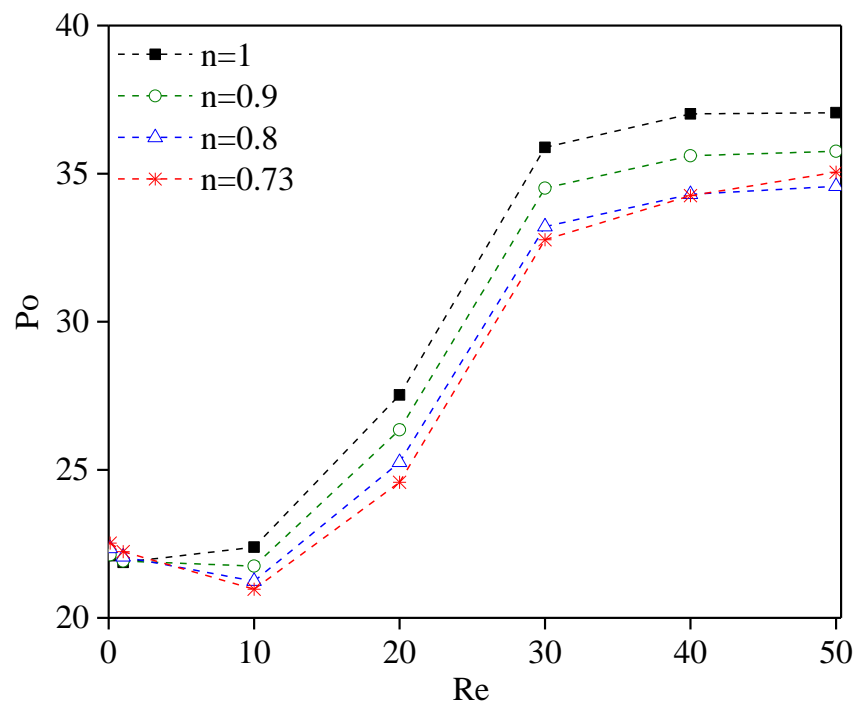

Figure 21. Variation of friction factor with the Reynolds numbers for different power-law indices.

\section{CONCLUSIONS}

Numerical simulations are performed to investigate the flow structure and mixing performance of shear-thinning fluids $(n<1)$ in a chaotic micromixer with two-layer serpentine crossing microchannels as a function of generalized Reynolds numbers ranging from 0.1 to 50 . The proposed mixer is of small size, millimeter size, which facilitates its insertion in different industrial applications. The study of the dynamic behavior of the flow shows the existence of hyperbolic points in the flow even at low Reynolds numbers, of the order of 0.1 . This reflects the sensitivity of this flow to the initial conditions and subsequently, the flow is considered chaotic. The mixing is quantified by a parameter called mixing index MI. The MI index is bounded by two values, 0 and 1 . For perfect mixing, MI tends to 1 . In our micromixer, this criterion exceeds $96 \%$ for all the simulations considered in this study. So our system is of great competences even at the creeping flow (Reynolds very low). The pressure losses, presented by the Poiseuille number, evaluated in this micromixer 
do not really present a disadvantage because they are, in the worst cases, less important than those measured in a straight tube in the case of an established flow.

\section{REFERENCES}

[1] Chafidz A, Rizal M, Faisal RM, Kaavessina M, Hartanto D, AlZahrani SM. Processing and properties of high density polyethylene/date palm fiber composites prepared by a laboratory mixing extruder. Journal of Mechanical Engineering and Sciences. 2018; 12(3): 3771-3785.

[2] Tran M, Memon Z, Saieed A, Pao W, Hashim F, Numerical simulation of two-phase separation in T-junction with experimental validation. Journal of Mechanical Engineering and Sciences. 2018; 12(4): 4216-4230.

[3] Ghanema A, Lemenand T, Valle DD, Peerhossaini H. Static mixers: Mechanisms, applications, and characterization methods - A review. Chemical engineering research and design, 2014; 92: 205-228.

[4] Cai G, Xue L, Zhang H, Lin J. A Review on Micromixers. Micromachines, 2017; 8(274): 1-27.

[5] Lee CY, Fu LM. Recent advances and applications of micromixers. Sensors and Actuators, 2018; B 259: 677-702.

[6] Nguyen NT, Wu Z. Micromixers - a review. Journal of Micromechanics and Microengineering, 2005; 15: 1-16.

[7] Ascanio G, Foucault S, Tanguy PA. Time-periodic mixing of shear-thinning fluids. Chemical Engineering Research and Design, 2004; 82(A9): 1199-1203.

[8] Lee CY, Wang WT, Liu CC, Fu LM. Passive mixers in microfluidic systems: A review. Chemical Engineering Journal, 2016; 288: 146-160.

[9] Niederkorn T, Ottino J. Chaotic mixing of shear-thinning fluids. AIChE journal, 1994; 40 (11): 1782-1793.

[10] Anderson PD, Galaktionov OS, Peters GWM, van de Vosse FN, Meijer HEH. Mixing of non-Newtonian fluids in time-periodic cavity flows. Journal of Non-Newtonian Fluid Mechanics, 2000; 93: 265-286.

[11] Choa CC, Chenb CL, Chena CK. Mixing enhancement of electrokinetically-driven non-Newtonian fluids in microchannel with patterned blocks. Chemical Engineering Journal, 2012; 191: 132-140.

[12] Afzal A, Kim KY. Flow and mixing analysis of non-Newtonian fluids in straight and serpentine microchannels. Chemical Engineering Science, 2014; 116: 263-274.

[13] Tsai RT, Wu CY, Chang CY, Kuo MY. Mixing Behaviors of Shear-Thinning Fluids in Serpentine Channel Micromixers. International Journal of Mechanical, Aerospace, Industrial, Mechatronic and Manufacturing Engineering, 2015; 9(7): 1329-1335.

[14] Shamsoddini R, Sefid M, Fatehi R. Incompressible SPH modeling and analysis of non-Newtonian power-law fluids, mixing in a microchannel with an oscillating stirrer. Journal of Mechanical Science and Technology, 2016; 30 (1): 307-316.

[15] Islami SB, Khezerloo M, Gharraei R. The effect of chaotic advection on mixing degree and pressure drop of non-Newtonian fluids flow in curved micromixers. Journal of the Brazilian Society of Mechanical Sciences, 2016; 10: 1-19. 
[16] Islami SB, Khezerloo M. Enhancement of Mixing Performance of Non-Newtonian Fluids using Curving and Grooving of Microchannels. Journal of Applied Fluid Mechanics, 2017; 10 (1): 127-141.

[17] Xia HM, Wan SYM, Shu C, Chewa YT. Chaotic micromixers using two-layer crossing channels to exhibit fast mixing at low Reynolds numbers. The Royal Society of Chemistry, 2005; 5: 748-755.

[18] Hossain S, Kim KY. Parametric investigation on mixing in a micromixer with twolayer crossing channels. SpringerPlus, 2016;5(794): 1-16.

[19] Hossain S, Lee I, Kim SM, Kim KY. A micromixer with two-layer serpentine crossing channels having excellent mixing performance at low Reynolds numbers. Chemical Engineering Journal, 2017; 327: 268-277.

[20] Srisamran C, Devahastin S. Numerical simulation of flow and mixing behavior of impinging streams of shear-thinning fluids. Chemical Engineering Science, 2006; 61: 4884-4892.

[21] Fellouah H, Castelain C, Ould-El-Moctar A, Peerhossaini H. The Dean instability in power-law and Bingham fluids in a curved rectangular duct. Journal of NonNewtonian Fluid Mechanics, 2010; 165: 163-173.

[22] Pinho FT, Whitelaw JH. Flow of non-Newtonian fluids in a pipe. Journal of NonNewtonian Fluid Mechanics, 1990; 34: 129-144.

[23] Das T, Chakraborty S. Recent trends and future challenges. Biomicrofluidics, 2009; Sadhana34(4): 573-590.

[24] Metzner AB, Reed JC. Flow of non-Newtonian fluids: correlation of the laminar, transition, and turbulent-flow regions. American Institute of Chemical Engineers Journal, 1955; 1(4): 434-440.

[25] Delplace F, Leuliet JC. Generalized Reynolds number for the flow of power law fluids in cylindrical ducts of arbitrary cross-section. The Chemical Engineering Journal, 1995; 56: 33-37.

[26] Ottino JM. Mixing, Chaotic advection, and turbulence. The Annual Review of Fluid Mechanics, 1990; 22: 207-53.

[27] Rohsenow WM, Hartnett JP, Cho YI. McGraw-Hill, Handbook of Heat Transfer, 3rd Ed. non-Newtoninan fluids, 1998, p 1-53.

[28] Hossain S, Kim KY. Mixing analysis in a three-dimensional serpentine split-andrecombine micromixer. Chemical Engineering Research and Design, 2015; 100: 95103.

[29] Raza W, Hossain S, Kim KY. Effective mixing in a short serpentine split-andrecombination micromixer. Sensors and Actuators B: Chemical, 2018; 258: 381-392.

[30] He M, Li W, Zhang M, Zhang J. Numerical investigation on the efficient mixing of overbridged split-and-recombine micromixer at low Reynolds number. Microsystem Technologies, 2019; 25(9): 3447-3461. 